\title{
Soft Computing Based Decision Making Approach for Tumor Mass Identification in Mammogram
}

\author{
Mahua Bhattacharya ${ }^{1^{\star}}$ and Arpita Das ${ }^{2}$ \\ ${ }^{* 1}$ Indian Institute of Information Technology \& Management, Morena Link Road, Gwalior-474003, India, e- \\ mail: mb@iiitm.ac.in \\ ${ }^{2}$ Institute of Radio Physics \& Electronics, University of Calcutta, 92, A.P.C. Road, Kolkata-700009, e-mail: \\ arpita.rpe@caluniv.ac.in
}

\begin{abstract}
An intelligent computer aided diagnosis system can be very helpful for radiologist in detecting and diagnosing breast cancer faster than typical screening program. This study attempted to segment the masses accurately and distinguish malignant from benign masses. The suspicious location of the breast masses are specified by the radiologists and then masses are accurately segmented using fuzzy c-means clustering technique. Fourier descriptors are utilized for the extraction of shape features of mammographic masses. These shape features along with the texture features are fed to the input of the ANFIS classifier for determination of the masses as benign, lobular or malignant. The classification system utilizes a simple Euclidian distance metric to determine the degree of malignancy. The study involves 40 digitized mammograms from MIAS, BIRADS database and has to be found $87 \%$ correct classification rate.
\end{abstract}

Keyword: Breast Cancer Diagnosis system, Breast Masses, Fourier Descriptors, Textural Descriptors, Fuzzy c-means Clustering, ANFIS model.

\section{Introduction}

Breast cancer is one of the leading causes of death for women. Women have better chance to survive if breast cancer can be detected early. XRay mammography is the most important modality, which is used for early detection of breast cancer $[1,3,6,7,10,13,15,19]$. The failure to detect any abnormal lesion at an early stage may lead to disastrous consequences. The mammographic images show signs of obstruction and many direct and indirect radiographic signs due to space occupying lesions in the tissue region of breast [1-3]. The improvement of mammographic image quality is essential for breast cancer screening. Therefore need exists to automate the process of analyzing a large number of mammograms and to discriminate the benign lesion from malignant one for proper therapy planning. The interpretation of mammograms by radiologists is done by visual examination of films to find out the presence of abnormalities. The computer aided diagnostics and detection procedures of masses as well as tissues are depicted by researchers in [4, 5, 15, $20,26,28,29]$ using digital mammogram and other modalities. The present work is continuation of our earlier work based on theory of shape [12-14] related to gradation of benignancy of tumor mass in tissue region. The concept of symmetry analysis of shape computes a distance function $D$ between the contour of the tumor model and the contour of the pattern tumor lesion. The distance function $D$ has been further utilized to classify the tumors mainly in two broad categories benign and malignant transformations. The shape of a contour was described on the basis of its structural features using chain code representation. The biological characteristics of tumor mass have been stated in [11] that the low grade homogeneous benign glioma is usually smooth masses with single radius whereas malignant transformation has multiple protrusions. We have suggested [12-14] earlier shape similarity measure $\mu$ to find out the prognosis of diseases where the idea of shape similarity measure has been implemented by minimization of distance function $D$ between the contours of tumor lesions and the model. These measurements give the indication of benignancy or malignancy of tumor lesions from a conventional coarse grading to a finer grading. In recent years, considerable efforts have been taken to develop automated methods for detection and classification of mammographic masses [7-10, 15-16, [18-23, 25, 27-28]. Brzakovic et al. investigated an expert system for analysis of mammogram [15]. Bruce et. al. [17] reported the results of applying multiresolution techniques to the problem of tumor mass classification. They utilized discrete wavelet transform modulus-maxima method for the extraction of mammographic mass shape features. In many breast cancer diagnostic systems fully automated techniques for mass segmentation is a major challenge. Several investigators exploited methods using intensity values to decide whether a pixel may belong in the region of interest $(\mathrm{ROI})$ or background [1819]. Mudigonda et al. [20] proposed an algorithm to segment masses by establishing intensity links, and to analyze oriented flow-like textural information in the ribbons of pixels across the margins of masses to determine if the segmented regions are true mass regions or false positives. $\mathrm{Li}$ et al. [21] developed a segmentation method that uses probability to determine segmented contours. Sahiner et. al. [22] developed an automated, three-stage segmentation method including clustering, active contour, and speculation detection stages. The method has 
been used for classification of the segmented masses as benign or malignant using speculation measures and morphological features. The approach based on support vector machine has been reported in [24] for detection of microcalcification in breast. In present paper authors have described an improved segmentation process of tumor mass using Fuzzy c-means clustering algorithm. In the next stage classifier has been designed using adaptive neuro fuzzy techniques [23, 25, 27] to discriminate the benignancy from malignancy of growth of tumor lesion in breast identified by mammograms. This method also incorporates the automated false positive reduction of mass boundaries. In this article, to describe the margins of tumor masses very precisely, we introduce Fourier descriptors [25] as shapebased features. To achieve high level of accuracy in malignancy detection, we combine statistical texture features of suspicious region in conjunction with shape-based features.

\section{Proposed Method for Classification of Tumor Masses}

Masses in mammograms are compact areas that appear brighter than the tissue in which they are embedded because of higher attenuation of $X$ rays. When the tissue surrounding a mass is fatty, detection is relatively easy. However, when a mass is buried in dense tissue, it may be very difficult to identify. The primary features that indicate malignancy are related to the mass size, shape, borders and texture. In present paper we have proposed techniques based on neuro fuzzy softcomputing to classify the tumor masses appearing in breast in different groups of benignancy and malignancy. Shape based boundary features and texture features have been extracted from region of interest $\mathrm{ROI}$ of tumor mass and which are fed to the classifier developed using adaptive neuro fuzzy based techniques. The classifier takes the decision, whether the masses are benign or malignant. The features extracted from shape and texture information provide much more accurate decision on benignancy/malignancy of masses than a single feature. The overview of the proposed method is presented below in Fig. (1).

\section{Segmentation of Tumor Mass Using Fuzzy c- Means Clustering Algorithm}

Segmentation of tumor mass is an important prior step for further classification/ identification. The radiologists may be confused due to the presence of high-contrast fibroglandular [29-30] tissue in the mammograms as the actual calcified masses. In this regard our objective is to develop a robust technique for segmentation of calcified masses from breast tissue. Presently fuzzy cmeans clustering algorithm has been used for intensity based segmentation of masses. Total number of fuzzy cluster centers chosen is three as shown in Fig. (2). Cluster center $A$ represents the healthy breast tissue. Second cluster $B$ represents false presence of mass region and $C$ represents actual mass region. Three cluster centers are selected to segment the tiny calcifications/tumors of mammograms in presence of different types of tissues and muscles. The accuracy of final classification of tumors in different grades of benignancy / malignancy depends on the superiority of segmentation process. In the proposed method, fuzzy c-means clustering algorithm has been used for intensity based segmentation of microcalcification clusters. Consulting with radiologists and after a detailed discussion with them it can be concluded that choice of three fuzzy cluster centers (as shown in Fig. 2) satisfy our requirements i.e. suppress the high FP rate and preserve the true segmentation details of fine calcification spots. In Fig. (2), cluster $A$ represents the normal breast tissues. Second cluster $B$ represents the false presence of microcalcifications and $C$ represents the actual calcification points. The segmentation results also justify our assumption. The ultimate Fuzzy partition membership functions have been shown in Fig. 2, which shows that there is an overlapping between the membership functions $A, B \& C$. If the possibility of belongingness of any region of breast to the calcification part is greater than $50 \%$ i.e. the membership value of curve $C>0.5$ (hashed portion of curve $\mathrm{C}$ ), decision may be taken that the particular region is within the calcified lesion. According to the decision rule, the shaded region in Fig. 2 indicates the Region of interest (ROI). Algorithm: Let $X=\left\{x_{1}, x_{2}, \ldots \ldots ., x_{n}\right\}$ be a set of given data. $A$ fuzzy $c$-partition of $X$ is a family of fuzzy subsets of $X$, denotes by $P=\left\{A_{1}, A_{2}, \ldots \ldots ., A_{c}\right\}$, which satisfies

$$
\sum_{i=1}^{C} A_{i}\left(x_{k}\right)=1
$$

The performance index of a fuzzy partition $P, \mathrm{~J}_{\mathrm{m}}$ $(P)$, is defined in terms of the cluster centers by the formula

$$
\mathrm{J}_{\mathrm{m}}\left(A, v_{l}, \cdots-v_{c}\right)=\sum_{\mathrm{k}=1} \underset{j}{\sum} \sum_{\mathrm{j}=1}\left[\mathrm{~A}_{\mathrm{i}}\left(\mathrm{x}_{\mathrm{k}}\right)\right]^{\mathrm{m}}\left\|\mathrm{x}_{\mathrm{k}}-\mathrm{v}_{\mathrm{i}}\right\|^{2}
$$

Where $\left\|x_{k}-v_{i}\right\|^{2}$ represents the distance between $x_{k}$ and $v_{i}$ ( $v i$ is the cluster centers). Clearly, the smaller the value of $\mathrm{J}_{\mathrm{m}}(P)$, the better the fuzzy partition $P$. Thus, the goal of fuzzy cmeans clustering method is to find a fuzzy partition $P$ that minimizes the performance index $\mathrm{J}_{\mathrm{m}}(P)$, which offers 


$$
\mathrm{v}_{\mathrm{i}}=\frac{\sum_{\mathrm{k}=1}^{\mathrm{N}}\left[\mathrm{A}_{\mathrm{i}}\left(\mathrm{x}_{\mathrm{k}}\right)\right]^{\mathrm{m}} \mathrm{x}_{\mathrm{k}}}{\sum_{\mathrm{k}=1}^{\mathrm{n}}\left[\mathrm{A}_{\mathrm{i}}\left(\mathrm{x}_{\mathrm{k}}\right)\right]^{\mathrm{m}}}
$$

\section{Extraction of boundary as feature using Fourier Descriptors}

Feature selection is the choice of descriptors in a particular application. In present work we have introduced Fourier descriptors as the boundary features [25] which have information regarding shape and margin of the segmented masses. Conventional shape descriptors like compactness, area, determination of number of concavity and convexity points may not always provide sufficient evidences to discriminate tumor masses of various characteristics and of heterogeneous structures. In present work we have proposed method of boundary description using Fourier descriptors to differentiate tumor masses of various characters very precisely. Algorithm: Let us consider a figure that describes $\mathrm{k}$-points digital boundary in the $x-y$ plane, staring at an arbitrary point $\left(\mathrm{x}_{0}, \mathrm{y}_{0}\right)$ to the coordinate pairs $\left(\mathrm{x}_{1}, \mathrm{y}_{1}\right),\left(\mathrm{x}_{2}, \mathrm{y}_{2}\right), \ldots,\left(\mathrm{x}_{\mathrm{k}-1}, \mathrm{y}_{\mathrm{k}-1}\right)$ along the boundary. These co-ordinates are represented by the form $x(k)=x_{k}$ and $y(k)=y k$. Thus the boundary can be represented as

$$
s(\mathrm{k})=[\mathrm{x}(\mathrm{k}), \mathrm{y}(\mathrm{k})] \quad \text { for } \mathrm{k}=0,1,2, \ldots \ldots, \mathrm{k}-1
$$

Each co-ordinate pair can be treated as a complex number so that

$$
\mathrm{s}(\mathrm{k})=\mathrm{x}(\mathrm{k})+\mathrm{j}^{*} \mathrm{y}(\mathrm{k}) \quad \text { for } \mathrm{k}=0,1,2, \ldots ., \mathrm{k}-1
$$

The $x$-axis is treated as real axis and $y$-axis as the imaginary one. The discrete Fourier transform (DFT) of $s(k)$ is given below

$$
\begin{aligned}
& \mathrm{K}-1 \\
& a(u)=(1 / K) \times \sum s(\mathrm{l}) \times e^{-(22 \pi u k \mathbb{K})} \quad \text { for } u=0,1,2, \ldots \mathrm{K}-1 \\
& k=0
\end{aligned}
$$

The complex coefficient $a(u)$ is the Fourier descriptor of the edge points along the boundary. Let us suppose that instead of all Fourier coefficients, only the first ' $P$ ' coefficients are used. This is equivalent to set $a(u)=0$ for $u>$ $(P-1)$. The overall global shape of the image has been identified. Thus only $50 \%$ of total Fourier descriptors are used for the representation of a boundary.

\section{Searching of feature subset using FCM}

The feature selection is an important issue in machine learning, which consists of finding feature subset of input training as well as test patterns that enables to describe information required to classify any pattern. The boundary or margin detected of tumor mass based on Fourier descriptors, introduces a large number of feature vectors. Thus to classify different boundaries, a standard classifier requires large number of inputs, to train the classifier. As a result large number of training cycles and huge memory are also required to accomplish the task. But it is obvious that a complicated structure invites the problem of over learning and chances of misclassification. In proposed method, fuzzy cmeans clustering technique has been used to reduce the shape descriptors into four clusters. The objective of FCM is to partition the large shape descriptors such that the degree of association is strong for descriptors within the same cluster and weak for the descriptors in different clusters.

\section{Statistical Texture Feature extraction}

Texture information plays an important role in image analysis and understanding. It measures the relative smoothness, average uniformity of the images. Homogeneous benign mass posses smooth and uniform texture whereas heterogeneous malignant mass having multiple protrusions and non uniform texture [11]. Texture property of tumor mass is an indicator to determine the degree of malignancy / benignancy of lesion.

Let $\mathrm{z}$ be a random variable denoting the gray levels which has range of $(0, L-1)$ and let $p\left(z_{1}\right)$, $\mathrm{I}=0,1,2, \ldots \ldots,(\mathrm{L}-1)$, be the corresponding histogram ( where $\mathrm{L} \rightarrow$ number of distinct gray levels). The $n$-th moment of $z$ about the mean is given as

$$
\begin{gathered}
\mathrm{L}-1 \\
\mu_{\mathrm{n}}(\mathrm{z})=\sum_{\mathrm{I}=0}\left(\mathrm{Z}_{\mathrm{I}}-\mathrm{m}\right)^{\mathrm{n}} p\left(\mathrm{Z}_{\mathrm{I}}\right)
\end{gathered}
$$

where $m$ is the mean value of $z$,

$$
\mathrm{m}=\sum_{\mathrm{I}=0}^{\mathrm{L}-1} \mathrm{Z}_{\mathrm{I}} p\left(\mathrm{Z}_{\mathrm{I}}\right)
$$

The second moment [the variance $\sigma^{2}(z)=\mu_{2}(z)$ ] has particular importance in textural description. It is a measure of gray level contrast that can be used to establish the descriptors of relative smoothness. The third moment $(n=3)$ is a measure of the skewness of the histogram that is generally useful for determination of the degree of symmetry of histograms whether they are skewed to the left (negative value) or right (positive value). This gives a rough idea of whether the gray levels are biased towards the dark or light side of the mean. Another useful texture measurement based on histograms include a measure of uniformity $(U)$ given by 


$$
\mathrm{U}=\sum_{\mathrm{I}=0}^{\mathrm{L}-1} p^{2}\left(\mathrm{Z}_{\mathrm{I}}\right)
$$

From the measure of uniformity we can conclude that a smooth texture is much more uniform than a rough one. Average entropy is a measure of gray-level variability and is 0 for a constant image. It is defined as

$$
\mathrm{e}=-\sum_{\mathrm{I}=0}^{\mathrm{L}-1} p\left(\mathrm{Z}_{\mathrm{I}}\right) \log _{2} p\left(\mathrm{Z}_{\mathrm{I}}\right)
$$

Classification of features into benign or malignant group

The proposed method uses adaptive neuro fuzzy network for classification of features into benign and malignant groups. Present work is an extension of earlier work [12-14] where shape based methodologies has been used for gradation of benignancy / malignancy of tumor mass. A finer grading system was suggested by us who shows not only the class of tumor mass but the tendency of growth either towards benignancy or malignancy. Present technique of classification of tumor mass in breast is based on adaptive neuro fuzzy soft-computing approach and more robust in terms of uncertainty management present in the system. This technique leads to the development of more accurate decision making procedure to discriminate the benign mass from the malignant one. Adaptive neuro fuzzy model removes the limitations of conventional back propagation neural networks [27]. To adapt the network with ever-changing environments, hybrid-learning rule has been used.

\section{Adaptive neuro fuzzy architecture}

Fig. 3 illustrates the reasoning mechanism of the adaptive neuro fuzzy inference architecture for boundary detection and texture analysis of tumor masses, where nodes of the same layer have similar functions as described below. Layer 1: Every node I in this layer is an adaptive node with a node function

$$
\begin{aligned}
& O_{1, i}=\mu_{\mathrm{Ai}}(\mathrm{x}), \\
& \mathrm{O}_{1, \mathrm{i}}=\mu_{\mathrm{Bi}}(\mathrm{y}),
\end{aligned}
$$

$$
\text { for } i=1,2
$$

Where $x$ (or $y$ ) is the input to node $i$ and $A_{i}\left(\right.$ or $\left.B_{i}\right)$ is a linguistic label (such as large or small) associated with this node. In other words $\mathrm{O}_{1, \mathrm{i}}$ is the membership grade of fuzzy set $A\left(A_{1}, A_{2}\right)$ or $B$ $\left(B_{1}, B_{2}\right)$. Here the membership function for $A$ can be any appropriate parameterized membership function, such as generalized bell function:

$$
\mu_{\mathrm{A}}(\mathrm{x})=\frac{1}{1+\left|\left(\mathrm{x}-\mathrm{c}_{\mathrm{i}}\right) / \mathrm{a}_{\mathrm{i}}\right| 2 \mathrm{~b}}
$$

Where $\left\{a_{i}, b_{i}, c_{i}\right\}$ is the parameter set. As the values of these parameters change, the bellshaped function varies accordingly. Parameters of this layer are referred to as premise parameters. Layer 2: Every node in this layer is a fixed node labeled $\Pi$, whose output is the product of all the incoming signals:

$$
\mathrm{O}_{2, \mathrm{i}}=\mathrm{w}_{\mathrm{i}}=\mu_{\mathrm{Ai}}(\mathrm{x}) \mu_{\mathrm{Bi}}(\mathrm{y}), \quad \mathrm{i}=1,2
$$

In general, any T-norm operator that performs fuzzy AND can be used as the node function in this layer. Layer 3: Every node in this layer is a fixed node labeled $N$. The $i^{\text {th }}$ node calculates the ratio of the rule's firing strength to the sum of all rules' firing strengths:

$$
\mathrm{O}_{3, \mathrm{i}}=\mathrm{w}_{\mathrm{i}}=\mathrm{w}_{\mathrm{i}} /\left(\mathrm{w}_{1}+\mathrm{w}_{2}\right), \quad \mathrm{i}=1,2 .
$$

For convenience, outputs of this layer are called normalized firing strengths. Layer 4: Every node $\mathrm{i}$ in this layer is an adaptive node with a node function

$$
\mathrm{O}_{4, \mathrm{i}}=\overline{\mathrm{w}}_{\mathrm{i}} \mathrm{f}_{\mathrm{i}}=\overline{\mathrm{w}}_{\mathrm{i}}\left(\mathrm{p}_{\mathrm{i}} x+\mathrm{q}_{\mathrm{i}} \mathrm{y}+\mathrm{r}_{\mathrm{i}}\right)
$$

Where $w_{i}$ is a normalized firing strength from layer 3 and $\left\{p_{i}, q_{i}, r_{i}\right\}$ is the parameter set of this node. Parameters of this layer are referred to as consequent parameters. Layer 5: The single node in this layer is fixed node labeled $\sum$, which computes the overall output as the summation of all incoming signals:

$$
\text { Overall output }=\mathrm{O}_{5,1}=\sum_{\mathrm{i}} \mathrm{W}_{\mathrm{i}} \mathrm{f}_{\mathrm{i}}=\sum_{\mathrm{i}} \mathrm{W}_{\mathrm{i}} \mathrm{f}_{\mathrm{i}} / \sum_{\mathrm{i}} \mathrm{W}_{\mathrm{i}}
$$

Hybrid Learning Rule: Training Algorithm of adaptive neuro fuzzy inference model

Hybrid leaning rule combines steepest decent method and least-squares estimator for fast identification of parameters in adaptive neuro fuzzy model. For hybrid learning to be applied in a batch mode, each epoch is composed of a forward pass and a backward pass. In the forward pass, after an input vector is presented, node outputs go forward until layer 4 and consequent parameters are identified by the least squares method. In the backward pass, the error signals propagate backward and the premise parameters are updated by gradient decent. The hybrid method converges much faster than a single approach since it reduces the search space dimensions of the original pure back propagation learning. Also the hybridization of neuro-fuzzy approaches is robust and adaptive 
even in the noisy, uncertain environment. Table-1 summarizes the activities in each pass. The limitation of adaptive neuro fuzzy model is that the architecture is learned well only when number of inputs are very small ( 3 to 4 only). In present paper we set the input feature vector size to 4 only and there are two bell-shaped membership functions which are assigned for each input variable. Thus number of fuzzy if-then rules for classifier learning is $2^{4}=16$ only. In conventional fuzzy inference system, the number of fuzzyrules is determined by an expert familiar with the target system to be modeled. In present simulation process, however no expert is available and number of membership functions assigned to each input variable is chosen empirically that is simply by trial and error. For present method there is no need to determine the minimal number of hidden units required to achieve a desired performance level.

\section{Decision making logic}

Design of appropriate decision rule is a crucial task for successful pattern recognition problem to classify a pattern accurately. In present paper, the classification of mammographic masses has been performed on the basis of both shape and texture based features of the patterns. The proposed neuro-fuzzy model for classification has been trained with twenty smooth textured, round shaped benign masses ("training set") specified by the radiologists. Then a set of 200 different mammograms from MIAS, and other databases (from EKO X-Ray and Imaging Institute, Calcutta) have been used to test the functionality of the proposed algorithm. Basically the indistinct and non-obvious tumor cases are studied in present work. The ultimate decision on the prognosis of 'test' tumor might be either benign or malignant but initially it was unpredictable.

For the classification process a distance function $\left(\mu_{1}\right)$ has been defined for determination of deviation of the mass from roundness and then to determine the degree of malignancy. Malignancy rate is higher for higher value of $\mu_{1}$. Next step is to determine the textural complexion of the test masses. For this purpose another distance function $\left(\mu_{2}\right)$ has been described for determination of degree of relative smoothness. Adaptive neuro fuzzy model has been trained with very smooth textured, round shaped masses and degree of benignancy/malignancy of the test masses has been determined with the help of these two distance functions. The distance function $\left(\mu_{1}\right)$ is the Euclidean distance and corresponding decisions are given below:

$$
\mu_{1}=|| \mathbf{D}_{1}-\mathbf{O}_{1}||
$$

Where, $\quad D_{1}=$ Desired output value for benign mass, $\mathrm{O}_{1}=$ output value of the test mammograms

$$
\begin{gathered}
\therefore \mu_{1}=\left[\left(D_{1}-0_{1}\right)^{\mathrm{T}}\left(\mathbf{D}_{1}-\mathbf{O}_{1}\right)\right]^{0.5} \\
=\left[\left(\mathrm{D}_{1}-\mathrm{O}_{1}\right)^{2}\right]^{0.5}
\end{gathered}
$$

The decisions based on boundary feature of test masses are defined below:

If $\mu_{1}<=20$ (threshold value), the shape \& margin of test masses are considered as: almost round or round to oval shape \& smooth boundary benign

If $20<=\mu_{1}<=40$ (threshold value), the shape \& margin of test masses are considered as: lobulated shape \& non-circumscribed boundary tendency towards malignancy

If $\mu_{1}>40$ ( threshold value), the shape \& margin of the test masses are considered as: irregular shape \& ill-defined boundary - malignant

Similarly,

$$
\mu_{2}=|| \mathbf{D}_{2}-\mathbf{O}_{2}||
$$

Where, $D_{2}=$ Desired output value for smooth benign mass, $\mathrm{O}_{2}=$ output value of the test mammograms.

$$
\begin{gathered}
\therefore \mu_{2}=\left[\left(\mathbf{D}_{2}-\mathrm{O}_{2}\right)^{\mathrm{T}}\left(\mathbf{D}_{2}-\mathbf{0}_{2}\right)\right]^{0.5} \\
=\left[\left(\mathrm{D}_{2}-\mathrm{O}_{2}\right)^{2}\right]^{0.5}
\end{gathered}
$$

The decisions based on texture feature of test masses are defined below:

If $\mu_{2}<=15$, textural complexion of test masses considered as: smooth

If $15<=\mu_{2}<=30$, textural complexion of test masses considered as:

\section{Tendency towards roughness}

If $\mu_{2}>30$, textural complexion of test masses considered as: coarse

The final decision on degree of benignancy / malignancy based on both boundary and texture features:



\section{Experimental Results}

We have implemented the proposed algorithm to databases (MIAS and BIRADS) consisting of 200 images. The classifier was first trained with obvious benign masses as identified by the expert radiologists. Other non-obvious cases have been tested and classified during the experiment. Our objective is to classify the test masses/calcifications and then to find out their belongingness either in benignancy or malignancy. For the classification process, a distance function $\left(\mu_{1}\right)$ has been defined to determine the roundness deviation of test tumors w.r.t trained benign tumor followed by the decision on the degree of malignancy. Malignancy rate is higher for higher value of $\mu_{1}$. Similarly another distance function $\left(\mu_{2}\right)$ has been described to determine the degree of relative smoothness of test tumors w.r.t trained benign tumor. In this case also the malignancy rate is higher for higher value of $\mu_{2}$. From the opinion of radiologists about the gradation of calcifications/masses into benign/malignant groups, we have defined the threshold margins of above mentioned distance functions $\mu_{1}$ and $\mu_{2}$. The final decisions on benignancy/malignancy gradation of indistinct tumors also support the pre-assumed threshold values. Few of the nonobvious case studies and final decision on benignancy / malignancy are given below:

Final decision on degree of malignancy of the segmented masses:

Data [1] $\rightarrow$ Tendency towards Malignant Stage,

Data [2] $\rightarrow$ Possibly in Malignant Stage,

Data [3] $\rightarrow$ Benign Stage,

Data [4] $\rightarrow$ Tendency towards Malignant Stage,

Data [5] $\rightarrow$ Possibly in Malignant Stage,

The detailed decision on degree of malignancy is described in Table-II.

Final decision on degree of malignancy of the segmented masses

Data [6] $\rightarrow$ Possibly in Malignant Stage,

Data [7] $\rightarrow$ Possibly in Malignant Stage,

Data [8] $\rightarrow$ Possibly in Malignant Stage,

Data [9] $\rightarrow$ Tendency towards Malignant Stage

Data [10] $\rightarrow$ Tendency towards Malignant Stage,

The detailed decision on degree of malignancy is described in Table-II.

\section{Conclusion}

In proposed methodology for tumor classification using mammogram, authors have attempted to develop a technique based on adaptive neuro fuzzy model by extracting the features (i) boundary of the lesion or region of interest ROI and (ii) texture of the surface. This classification concerns the prediction regarding the prognosis of the disease either towards benignancy or malignancy using machine intelligence considering features like shape, margin and texture. The proposed classification network has been trained by hybrid-learning rule with maximum fifty epochs. The learning process is continued till the performance goal reaches its optimum level. The output node values indicate the deviation of the test masses with respect to the trained benign masses. It has been noted that there is no sharp boundary among the three stages: benign, tendency towards malignancy and malignant stage and the partition among these stages is also fuzzy partition. Under this circumstance the adaptive neuro fuzzy is a robust soft-computing approach to take decision even in the presence of uncertainty as it appears in the process of classification of tumor masses. In the proposed method, fuzzy c-means clustering algorithm has been used for intensity-based robust segmentation of tumor masses especially in the dense breast. The presence of false positive mass region has been reduced properly by choosing the cluster centers. The second cluster center actually containing with the false presence of mass region, which is suppressed in the final segmentation. In present paper, we have assumed that the structure of adaptive neuro fuzzy model is fixed for both shape and texture determination of masses. The premise parameters \& consequent parameters are identified in both the cases separately (using separate training dataset for each cases) by the hybrid learning rule.

\section{Acknowledgment}

The authors would like to thank to Dr. S. K. Sharma of EKO X-Ray and Imaging Institute, Kolkata.

\section{References}

[1] Carter M. M., McCook B. M., Shaff M. I, Lamballe A. K., Winfield A. C. (1987) Special report: Breast Imaging Applied Radiology.

[2] Homer M.J., Eliza R, Spellman P. (1986) Applied Radiology 16, 547-548.

[3] Pagami J.J., Bassett L.W., Gold R.H., Bendetti J., Arndt R.D., Linsman J., Scanlan R.L. (1985) Amer J.Roentgnol, 135, 843-845.

[4] Verma B., Zakos J. (2001) IEEE Trans. Information Technology in Biomedicine, 5(1), 46-54.

[5] Nishikawa R., Giger M., Doi K., Vyborny C., Schmidt R., (1994) State of the Art of Digital Mammographic Image Analysis. Singapore: World Scientific, 7, 82-102.

[6] Feig S. A., McLelland R.( 1983) New York: Masson.

[7] Kupinski M.A., Giger M.L. (1998) IEEE Trans. Med. Imaging, 17(4), 510-517.

[8] Magnin I. E., Alaoui M. El, Bremond A..(1989) Proc. SPIE, 1137, 170-175. 
[9] Davies D. H. Dance D. R. (1990) Phys. Med. Biol., 35(8), 1111-1118.

[10] Shen L., Rangayyan R. M., Desautels J. E. L. (1994) IEEE Trans. Med. Imag, 13, 263-274.

[11] Set M., Leith J. T. (1994) J. Thoer. Biology, 169, 327-328.

[12] Bhattacharya M., Dutta Majumder D. (2008) Pattern Directed Information Analysis (Algorithms, Architecture \& Applications ) publisher: New Age International Wiley, pp:454-486.

[13] Bhattacharya M. Dutta Majumder D. (1998) $16^{\text {th }}$ Int. CODATA (France) Conference, Delhi.

[14] Dutta Majumder D., Bhattacharya M. (2000) International Journal of Systems \& Cybernetes, MCB publications, UK, 29(7/8), 871-895.

[15] Brzakovic D., Luo X. M., Brzakovic P. (1990) IEEE Trans. Med. Imag., 9, 233241.

[16] Kilday J. Palmieri F., Fox M. D.( 1993) IEEE Trans. Med. Imag., 12, 664-669.

[17] Bruce L. M., Adhami R. R. (1999) IEEE Trans. Medical Imaging, 18(12), 11701177.

[18] Sahiner B, Chan H.P., Wei D, Petrick N, Helvie M.A., Adler D.D., Goodsit M.M. (1996) Medical Physics, 23(10), 16711684.

[19] Mendez A.J., Tahoces P.G., Lado M.J., Souto M., Vidal J.J.( 1998) Medical Physics, 25(6), 957-964.

[20] Mudigonda N. R., Rangayyan R.M., Desautels J. (2001) IEEE Trans. Med. Imaging, 20(12), 1215-1227.
[21] Li H., Wang Y., Liu KJR, Lo S-C, Freedman MT (2001) IEEE Transactions on Medical Imaging, 20( 4), 289-301.

[22] Sahiner B., Chan H.P., Petrick N., Helvie M.A., Hadjiiski L.M. (2001) Med. Physics, 28, 1455-1465.

[23] Hall L.O., Bensaid A.M., Clarke L.P., Velthuizen R.P., Silbiger M.S., Bezdek J.C. (1992) IEEE Trans. Neural Networks, 3( 5), 672-682.

[24] Galatsanos N. P., Nishikawa R. M., I. ElNaqa, Miles Y. Y., Wernick N. (2002) IEEE Trans. Medical Imaging, 21( 2), $1552-1563$.

[25] Bhattacharya M., Das A. (2006), Proc. International conference on Machine Vision and Image Processing (IMVIP06), Dublin University, Ireland, UK pp: $52-59$

[26] Jiang J., Yao B., Wason A.M.( 2007) Computerized Med. Imaging and Graphics, 31, 49-61.

[27] Jang J.-S. R. (1993) IEEE Trans. Systems, Man and Cybernetics, 23(3), 665-685.

[28] Abbey C. K., Zemp R. J., Liu J., Lindfors K. K., Insana M. F. (2006) IEEE Trans. Medical Imaging, 25(2), 198 -209.

[29] Klifa C., Gamio J. C., Wilmes L., Laprie A., Lobo C., DeMicco E., Watkins M., Shepherd J., Gibbs J., Hylton N.( 2004) Proc. of the 26th Annual International Conf. of the IEEE EMBS, San Francisco, USA.

[30] Chen S., Zhang D. (2004) IEEE Trans. Systems, Man, And Cybernetics-Part B: Cybernetics, 34(4), 1907- 1916. 
Table I: Two passes in the hybrid learning procedure adaptive neuro-fuzzy inference

\begin{tabular}{|l|l|l|}
\hline$\#$ & \# Forward pass & \# Backward pass \\
\hline Premise parameters & Fixed & Gradient descent \\
\hline $\begin{array}{l}\text { Consequent } \\
\text { parameters }\end{array}$ & Least squares estimator & Fixed \\
\hline Signals & Node outputs & Error signals \\
\hline
\end{tabular}

Table II: Final decision to determine the degree of benignancy/malignancy of the tumor masses as identified in mammograms

\begin{tabular}{|l|l|l|l|l|l|}
\hline Database of Masses & $\begin{array}{l}\text { Distance } \\
\text { Function } \mu_{1}\end{array}$ & $\begin{array}{l}\text { Decision on Margin of } \\
\text { the Masses }\end{array}$ & Distance Function $\mu_{2}$ & $\begin{array}{l}\text { Decision on Texture of } \\
\text { the Masses }\end{array}$ & $\begin{array}{l}\text { Final } \\
\text { Degree of Malignancy }\end{array}$ \\
\hline Data1 & 21.5666 & $\begin{array}{l}\text { Dendency towards } \\
\text { malignancy }\end{array}$ & 3.2621 & Smooth & $\begin{array}{l}\text { ondency towards } \\
\text { malignant stage }\end{array}$ \\
\hline Data2 & 36.0873 & $\begin{array}{l}\text { tendency towards } \\
\text { malignancy }\end{array}$ & 25.4034 & $\begin{array}{l}\text { tendency towards } \\
\text { roughness }\end{array}$ & $\begin{array}{l}\text { possibly in malignant } \\
\text { stage }\end{array}$ \\
\hline Data3 & 18.4550 & benign & 17.8586 & $\begin{array}{l}\text { tendency towards } \\
\text { roughness }\end{array}$ & benign stage \\
\hline Data4 & 49.9834 & malignant & 13.4793 & Smooth & $\begin{array}{l}\text { tendency towards } \\
\text { malignant stage }\end{array}$ \\
\hline Data5 & 47.4203 & malignant & 29.3304 & $\begin{array}{l}\text { tendency towards } \\
\text { roughness }\end{array}$ & $\begin{array}{l}\text { possibly in malignant } \\
\text { stage }\end{array}$ \\
\hline Data6 & 49.5854 & malignant & 28.4788 & $\begin{array}{l}\text { tendency towards } \\
\text { roughness }\end{array}$ & $\begin{array}{l}\text { possibly in malignant } \\
\text { stage }\end{array}$ \\
\hline Data7 & 48.8889 & malignant & 32.4399 & Coarse & $\begin{array}{l}\text { possibly in malignant } \\
\text { stage }\end{array}$ \\
\hline Data8 & 49.9894 & malignant & 36.3679 & Coarse & $\begin{array}{l}\text { possibly in malignant } \\
\text { stage }\end{array}$ \\
\hline Data9 & 48.7158 & malignant & 10.9576 & $\begin{array}{l}\text { Smooth } \\
\text { Condency towards } \\
\text { malignant stage }\end{array}$ \\
\hline Data10 & Benign & 32.3870 & $\begin{array}{l}\text { Tendency towards } \\
\text { Malignant Stage }\end{array}$ \\
\hline
\end{tabular}

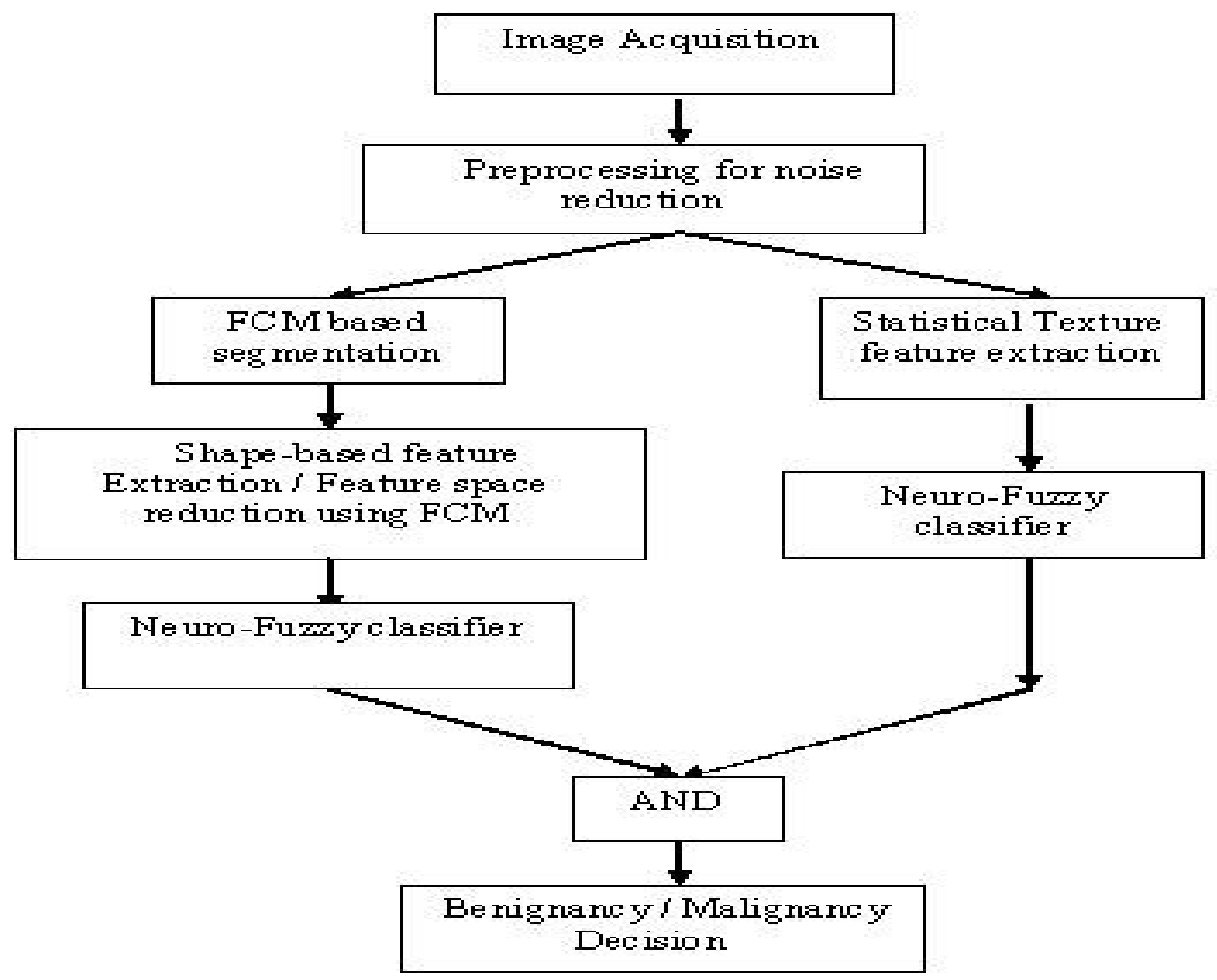

Fig. 1 Brief overview of present work 


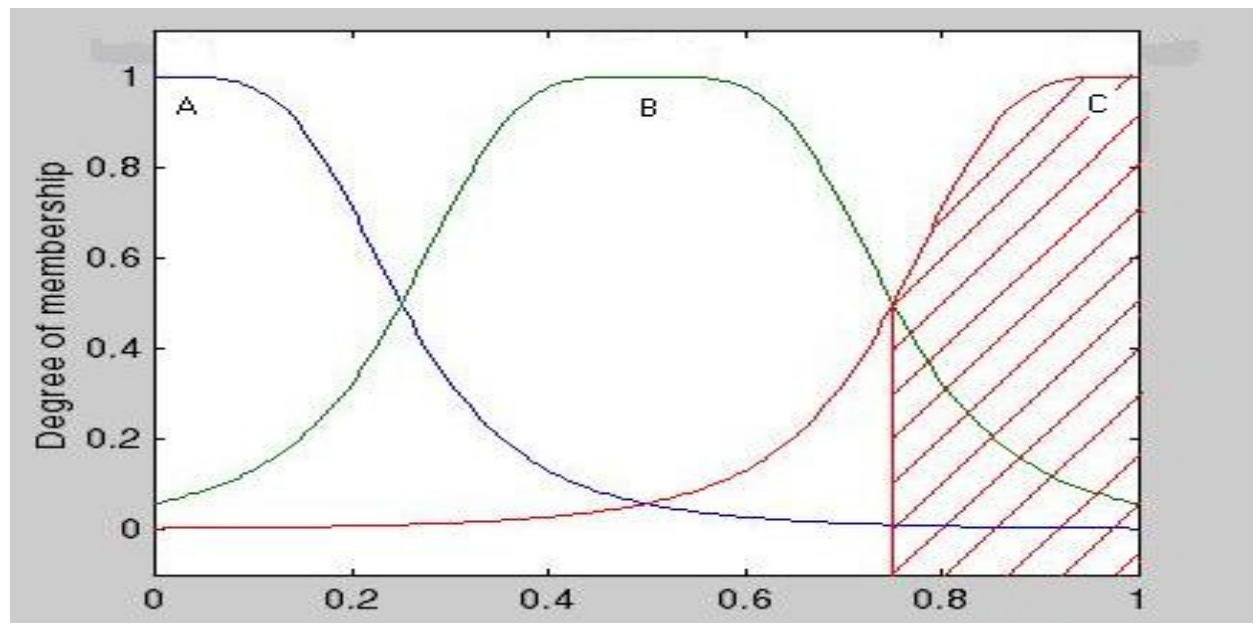

Fig. 2 Final Fuzzy Partition Membership Functions

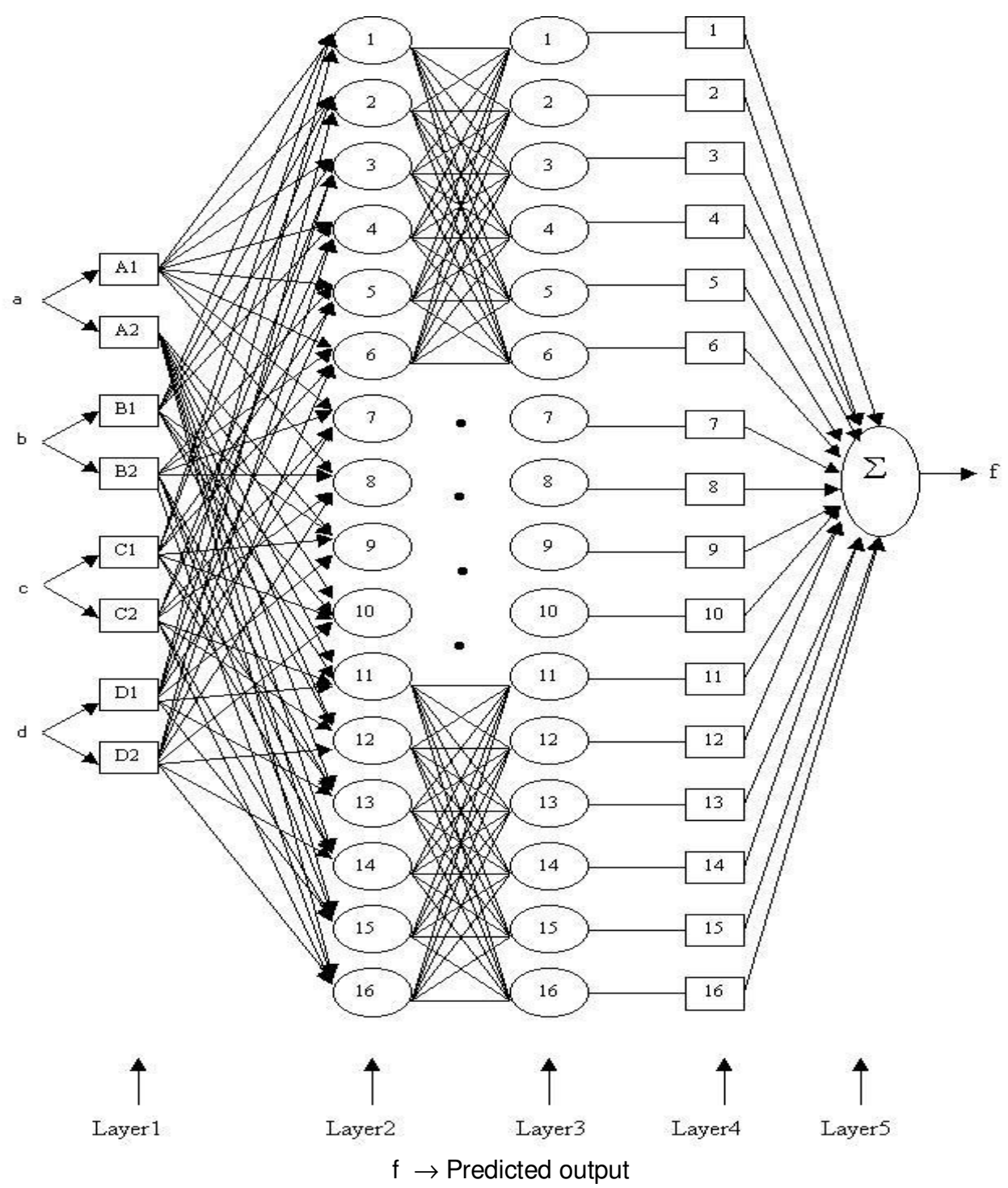

Fig. 3 The ANFIS Model for Final classification 
Accurate segmentation of non obvious test masses: (SET-I)

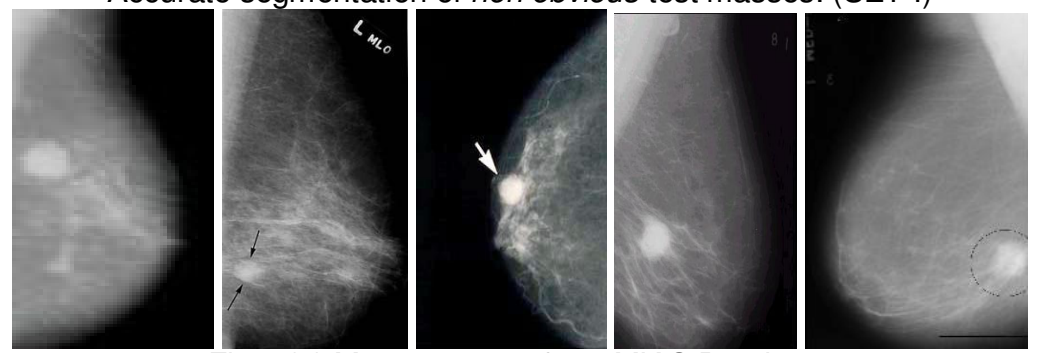

Fig. 4(a) Mammograms from MIAS Database
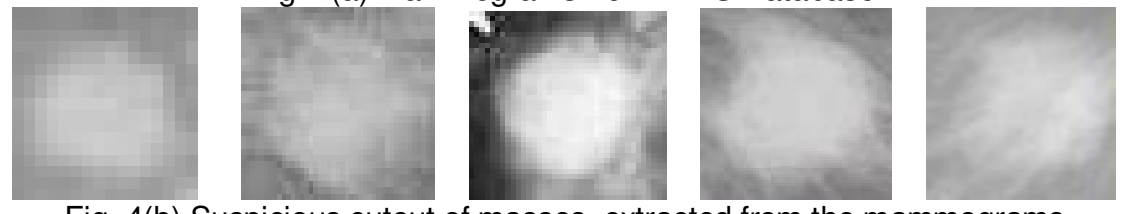

Fig. 4(b) Suspicious cutout of masses, extracted from the mammograms
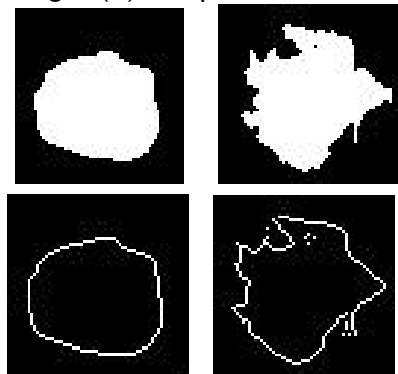

[1]


[2]



[3]

$[4$
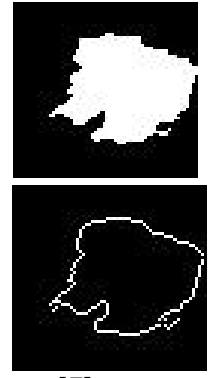

[5]

Fig. 4(c) Accurate contour segmentation of masses using FCM

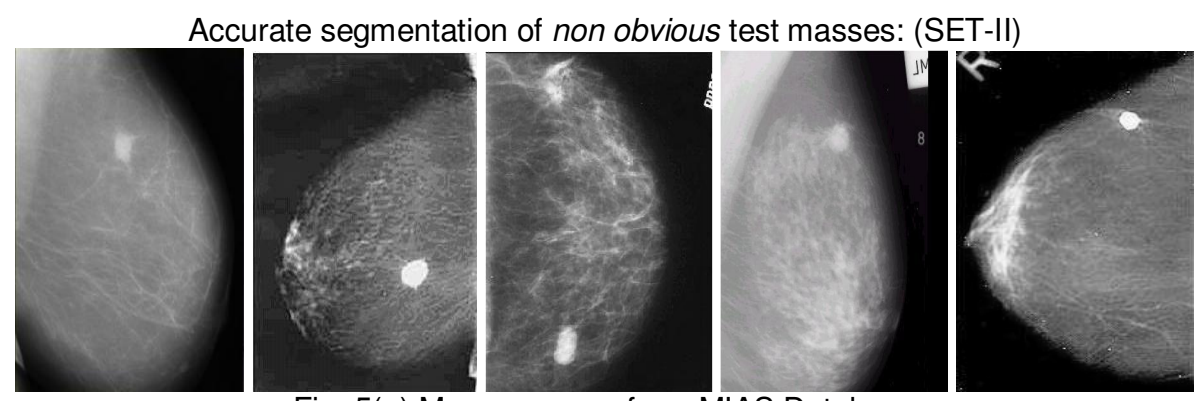

Fig. 5(a) Mammograms from MIAS Database
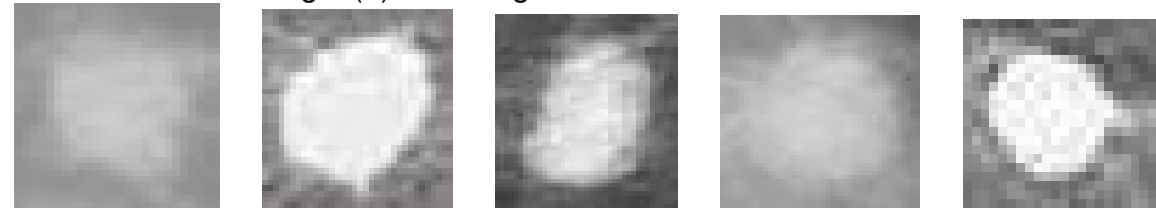

Fig. 5(b) suspicious cutout of masses, extracted from the Mammograms

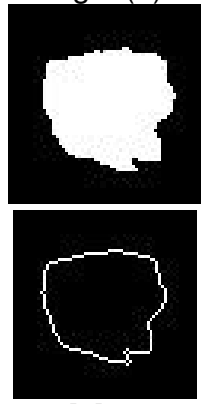

[6]
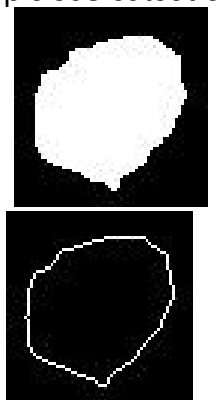

[7]
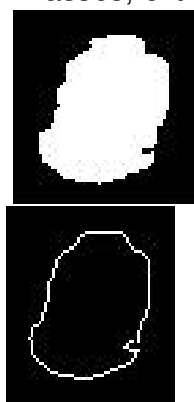

[8]
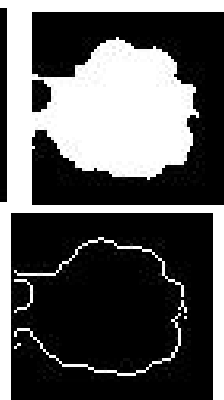

[9]
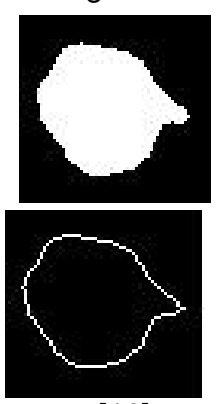

[10]

Fig. 5(c): Accurate contour segmentation of masses using FCM 